\title{
Padrões e comportamentos alimentares em crianças portadoras do Transtorno do
}

\section{Espectro Autista}

\author{
Eating patterns and behaviors in children with Autism Spectrum Disorder \\ Patrones y comportamientos alimentarios en niños con Trastorno del Espectro Autista
}

Recebido: 26/08/2021 | Revisado: 31/08/2021 | Aceito: 20/09/2021 | Publicado: 22/09/2021

\author{
Brenda Souza Mendes \\ ORCID: https://orcid.org/0000-0002-3095-6877 \\ Centro Universitário Santo Agostinho, Brasil \\ E-mail: melo25112@gmail.com \\ Jessyca Maria Lopes Freire \\ ORCID: https://orcid.org/0000-0002-0609-0161 \\ Centro Universitário Santo Agostinho, Brasil \\ E-mail: jessycafreire19@gmail.com \\ Daniele Rodrigues Carvalho Caldas \\ ORCID: https://orcid.org/0000-0001-8830-0283 \\ Centro Universitário Santo Agostinho, Brasil \\ E-mail:danielerccaldastrabalho@gmail.com \\ Tatiele Castelo de Oliveira \\ ORCID: https://orcid.org/0000-0002-1240-9140 \\ Centro Universitário Santo Agostinho, Brasil \\ E-mail:oliveirac.tatiele@gmail.com \\ Liejy Agnes dos Santos Raposo Landim \\ ORCID: https://orcid.org/0000-0002-8214-2832 \\ Centro Universitário Santo Agostinho, Brasil \\ E-mail: liejyagnes@gmail.com
}

\begin{abstract}
Resumo
Analisar os padrões e comportamentos alimentares em crianças portadoras do Transtorno do Espectro Autista (TEA). Trata-se de uma revisão integrativa da literatura. A busca dos artigos foi realizada nas bases de dados da BVS, Scielo, Lilacs, PubMED e Google Acadêmico entre os períodos de agosto de 2020 a junho de 2021. Foram encontrados 62 artigos e somente 11 foram selecionados para a discussão dos resultados. Os descritores utilizados foram: "Padrões Alimentares", "Comportamento Alimentar", e "Transtorno do Espectro Autista". Os critérios de inclusão foram artigos originais, indexados nas bases de dados selecionadas, nos idiomas: português e inglês; publicado entre os anos de 2011 e 2021. Os critérios de exclusão foram publicações de teses, anais de congressos, dissertações, monografias e artigos que não tinham aderência à temática. Quanto aos padrões alimentares as crianças preferem alimentos de alta densidade energética como as bebidas açucaradas e comidas crocantes, e apresentam um baixo consumo de frutas, vegetais, peixes e laticínios. Quanto aos comportamentos identificou-se uma grande recusa alimentar e relutância em comer novos alimentos como frutas, vegetais, peixes e laticínios, além de comportamentos problemáticos como resistência para sentar-se à mesa e comer com outras pessoas, entrar no refeitório e não ficarem sentados à mesa até a refeição acabar, e em aceitarem novas preparações, além disso ficou evidente que o comportamento dos pais influencia diretamente na seletividade alimentar e em suas escolhas. $\mathrm{O}$ acompanhamento dos pais de crianças com TEA trarão resultados positivos no entendimento desses padrões e comportamentos alimentares dessas crianças, para contribuir com os profissionais da área.
\end{abstract}

Palavras-chave: Crianças; Hábitos alimentares; Problemas de comportamento.

\begin{abstract}
To analyze eating patterns and behaviors in children with Autistic Spectrum Disorder (ASD).This is an integrative literature review. The search for articles was performed in the databases of the VHL, Scielo, Lilacs, PubMED and Academic Google between the periods of August 2020 to June 2021. 62 articles were found and only 11 were selected for discussion of the results. The descriptors used were: "Eating Patterns", "Eating Behavior", and "Autistic Spectrum Disorder". The inclusion criteria were original articles, indexed in the selected databases, in the languages: Portuguese and English; published between 2011 and 2021. Exclusion criteria were publications of theses, conference proceedings, dissertations, monographs and articles that did not adhere to the theme. Regarding dietary patterns, children prefer foods with high energy density such as sugary drinks and crunchy foods, and have a low consumption of fruits, vegetables, fish and dairy products. As for the behaviors, a great food refusal and reluctance to eat new foods such as fruits, vegetables, fish and dairy products were identified, in addition to problematic behaviors such as
\end{abstract}


resistance to sit at the table and eat with other people, enter the cafeteria and not sit at the table until the meal is over, and in accepting new preparations, it was also evident that the behavior of parents directly influences food selectivity and their choices. The monitoring of parents of children with ASD will bring positive results in the understanding of these children's eating patterns and behaviors, to contribute to professionals in the area.

Keywords: Children; Eating habits; Behavior problems.

\section{Resumen}

Analizar patrones y comportamientos alimentarios en niños con trastorno del espectro autista (TEA). Se trata de una revisión integradora de la literatura. La búsqueda de artículos se realizó en las bases de datos de la BVS, Scielo, Lilacs, PubMED y Academic Google entre los períodos de agosto de 2020 a junio de 2021. Se encontraron 62 artículos y solo se seleccionaron 11 para discusión de los resultados. Los descriptores utilizados fueron: "Patrones de alimentación", "Conducta alimentaria" y "Trastorno del espectro autista". Los criterios de inclusión fueron artículos originales, indexados en las bases de datos seleccionadas, en los idiomas: portugués e inglés; publicados entre 2011 y 2021. Los criterios de exclusión fueron publicaciones de tesis, actas de congresos, disertaciones, monografías y artículos que no se ajustaran a la temática. En cuanto a los patrones dietéticos, los niños prefieren alimentos con alta densidad energética como bebidas azucaradas y alimentos crujientes, y tienen un bajo consumo de frutas, verduras, pescado y productos lácteos. En cuanto a las conductas, se identificaron un gran rechazo a la comida y reticencia a comer nuevos alimentos como frutas, verduras, pescado y productos lácteos, además de conductas problemáticas como resistencia a sentarse a la mesa y comer con otras personas, entrar a la cafetería. y no sentarse a la mesa hasta que termine la comida, y al aceptar nuevas preparaciones, también fue evidente que el comportamiento de los padres influye directamente en la selectividad alimentaria y sus elecciones. El seguimiento de los padres de niños con TEA traerá resultados positivos en la comprensión de los patrones y comportamientos alimentarios de estos niños, para aportar a los profesionales del área.

Palabras clave: Niños; Hábitos alimenticios; Problemas de comportamiento.

\section{Introdução}

O autismo é um transtorno global do desenvolvimento conhecido como Transtorno do Espectro do Autismo (TEA). O TEA representa um conjunto diversificado e heterogêneo de estados unificados pela presença variável de comportamentos específicos (estereotipado e imutável e às vezes agressivo) que afetam as funções sociais e de comunicação (efeito social), interação social (isolamento), e falhas no desenvolvimento social e intelectual, que afeta o comportamento e repercute na vida social e na linguagem de seus portadores (Fernandes et al., 2016; Costa et al., 2020).

O TEA geralmente começa nos primeiros anos de vida, e manifesta-se em todas as etnias ou raças, grupos socioeconômicos, e gêneros. Comumente está associado a outros distúrbios ou condições médicas, como: déficit de atenção, hiperatividade, ansiedade, depressão, transtorno obsessivo-compulsivo (TOC), epilepsia e transtornos genéticos (Gomes et al., 2020).

Sua etiologia ainda é desconhecida, mas a tendência atual é tratá-la como uma síndrome multicausal, composta por fatores genéticos, neurológicos e sociais da criança. Estima-se que a prevalência global de TEA seja de aproximadamente 70 casos para cada 10.000 habitantes, sendo quatro vezes mais comum em meninos. No Brasil, apesar dos poucos estudos epidemiológicos que possam estimar melhor os dados nacionais, a taxa de incidência de autismo é de 27,2 casos para cada 10.000 habitantes (Pinto et al., 2016).

O diagnóstico é feito por avaliação clínica, com associação de critérios de diagnósticos específicos para o autismo. Não existem estudos laboratoriais que identifiquem a doença, cujas características mais comuns observadas em pacientes com Transtorno do Espectro Autista estão principalmente relacionadas ao comprometimento do desenvolvimento da linguagem e da interação social, no entanto, ainda existem alguns distúrbios gastrointestinais com a diminuição da produção de enzimas digestivas e inflamação da parede intestinal (Gomes et al., 2016).

A seletividade alimentar se destaca entre as mudanças comportamentais no TEA, e caracteriza-se pelo baixo apetite, recusa em comer e nenhum interesse pelo alimento. Essa combinação pode resultar em algumas limitações na variedade de alimentos consumidos, e manterá a resistência ao experimentar novos alimentos. Esta atitude pode muitas vezes ser transitória durante à fase de adaptação ao novo alimento ou perdurará durante todo o período de desenvolvimento da pessoa. Contudo, 
limitar as variedades na hora das refeições pode aumentar as deficiências nutricionais e prejudicar o organismo, pois a ingestão de macro e micronutrientes está intimamente relacionada ao consumo de energia e ao bom funcionamento do organismo (Westwood et al., 2017; Rocha et al., 2019).

Portanto, é de extrema importância analisar o comportamento alimentar do autista, tanto para identificar os principais transtornos alimentares e suas características, quanto os principais alimentos consumidos, a fim de contribuir para o avanço do conhecimento sobre o assunto, e discutir a importância do diagnóstico precoce dos Transtornos Alimentares do Espectro do Autismo, pois quanto mais rápido o diagnóstico, melhores os desfechos a longo prazo (Carvalho et al., 2013).

Desta maneira, este estudo tem como objetivo analisar os padrões e comportamentos alimentares em crianças portadoras do Transtorno do Espectro Autista.

\section{Metodologia}

Trata-se de uma revisão integrativa da literatura, de natureza qualitativa e exploratória, com abordagem teórica (Sousa et al., 2017). A pesquisa apresenta informações sobre padrões e o comportamento alimentar de crianças com TEA, para demonstrar a importância da discussão da temática, assim como, da colaboração dos pais que é essencial no âmbito da vida da criança.

Foi realizada uma busca das publicações/artigos, dos últimos 10 anos, utilizando cinco bases de dados eletrônicos, Biblioteca Virtual de Saúde da (BVS), Scientific Electronic Library Online (SCIELO), Literatura Latino Americana e do Caribe em Ciências da Saúde (LILACS), National Library of Medicine (PUBMED) e o Google Acadêmico, entre os períodos de agosto de 2020 a junho de 2021. Foram utilizados descritores nos idiomas português "transtorno do espectro autista", "Padrões Alimentares" e "comportamento alimentar"; e no idioma inglês "Autism spectrum disorder", "Food Standards" and “eating behavior" cadastrados nos descritores em Ciências da Saúde (DeCS).

As buscas foram realizadas no período de agosto de 2020 a junho de 2021, onde inicialmente foram selecionados 62 artigos a partir dos descritores. Os critérios de inclusão foram: artigo original, indexado nas bases de dados selecionadas, nos idiomas: português e inglês; publicado entre os anos de 2011 e 2021. A escolha desse período atenderá ao critério de temporalidade, em que se considerará o recorte de 10 anos, por se tratar de publicações mais atuais. Nos critérios de exclusão foram adotados: publicações de teses, anais de congressos e afins, dissertações, monografias e artigos que não tinham aderência à temáticas, indexados nas bases de dados selecionadas, nos idiomas português e inglês; publicados entre os anos de 2011 e 2021.

Após a seleção dos artigos ( $\mathrm{n}=11$, sendo 1 na língua portuguesa e 10 na língua inglesa) foi realizada a leitura e interpretação dos estudos cujas informações foram analisadas sistematicamente e agrupadas em um quadro, preenchido de acordo com o autor/ano, objetivo, amostra e considerações finais.

\section{Resultados e Discussão}

Foram selecionados artigos entre os anos de 2014 a 2021, onde a maioria adotou o método quantitativo de pesquisa, através de delineamento transversal observacional e descritivo, cujos objetivos buscaram descrever os padrões e comportamentos alimentares em crianças com TEA.

No Quadro 1 estão reunidos os principais resultados sobre os padrões e comportamentos alimentares em crianças portadoras do Transtorno do Espectro Autista (TEA). 
Quadro 1 - Distribuição dos resultados encontrados sobre os padrões e comportamentos alimentares em crianças portadoras do transtorno do espectro autista (TEA), publicados entre os períodos de 2014 a 2021 seguindo os autores, ano da publicação, objetivo, amostra da pesquisa, e considerações finais.

\begin{tabular}{|c|c|c|c|}
\hline AUTOR/ANO & OBJETIVO & AMOSTRA & CONCLUSÃO \\
\hline $\begin{array}{l}\text { Kral et al. } \\
\text { (2014) }\end{array}$ & $\begin{array}{l}\text { Comparar crianças com } \\
\text { Transtornos do Espectro do } \\
\text { Autismo (TEA) e crianças com } \\
\text { desenvolvimento típico (TDC) } \\
\text { em resultados relacionados ao } \\
\text { peso e relatado pelo cuidador } \\
\text { dos comportamentos } \\
\text { alimentares infantis e práticas } \\
\text { alimentares. }\end{array}$ & $\begin{array}{l}25 \text { crianças com TEA e } 30 \\
\text { com TDC, com as idades } \\
\text { de } 4 \text { a } 6 \text { anos. }\end{array}$ & $\begin{array}{l}\text { As crianças com TEA, } \\
\text { especialmente aqueles com } \\
\text { sensibilidade sensorial oral } \\
\text { atípica, estão em maior risco de } \\
\text { comportamentos de evitação de } \\
\text { alimentos e podem requerem } \\
\text { suporte adicional em vários } \\
\text { domínios de alimentação. }\end{array}$ \\
\hline $\begin{array}{c}\text { Sánchez et al. } \\
\text { (2015) }\end{array}$ & $\begin{array}{l}\text { Avaliar a relação entre a } \\
\text { presença de TEA em crianças e } \\
\text { adolescentes menores de } 18 \\
\text { anos e seletividade e consumo } \\
\text { alimentar. }\end{array}$ & 11 artigos & $\begin{array}{l}\text { A seletividade alimentar é mais } \\
\text { frequente em crianças e } \\
\text { adolescentes com TEA do que } \\
\text { naqueles com DT, o que } \\
\text { poderia influenciar seu estado } \\
\text { nutricional. Isso deve ser } \\
\text { levado em consideração após o } \\
\text { diagnóstico. }\end{array}$ \\
\hline $\begin{array}{c}\text { Stough et al. } \\
\text { (2015) }\end{array}$ & $\begin{array}{l}\text { Analisar os comportamentos na } \\
\text { hora das refeições associados ao } \\
\text { consumo de alimentos } \\
\text { desconhecido por crianças com } \\
\text { transtorno do espectro do } \\
\text { autismo. }\end{array}$ & $\begin{array}{l}\text { Crianças de } 2 \text { a } 8 \text { anos de } \\
\text { idade e com diagnóstico } \\
\text { de TEA. }\end{array}$ & $\begin{array}{l}\text { As intervenções clínicas para a } \\
\text { seletividade alimentar em } \\
\text { crianças com TEA podem } \\
\text { fornecer educação aos pais } \\
\text { sobre estratégias eficazes para } \\
\text { os pais durante as refeições e } \\
\text { diminuição r dos } \\
\text { comportamentos inadequados } \\
\text { das crianças durante as } \\
\text { refeições. }\end{array}$ \\
\hline $\begin{array}{l}\text { Castro et al. } \\
\text { (2016) }\end{array}$ & $\begin{array}{l}\text { Avaliar a ingestão dietética e } \\
\text { identificar problemas de } \\
\text { comportamento alimentar em } \\
\text { crianças e adolescentes do sexo } \\
\text { masculino com TEA quando } \\
\text { comparados a controles } \\
\text { pareados, bem como os } \\
\text { sentimentos dos pais ou } \\
\text { cuidadores sobre as estratégias } \\
\text { para lidar com problemas } \\
\text { alimentares. }\end{array}$ & Pacientes com TEA & $\begin{array}{l}\text { Destaca-se sobre a importância } \\
\text { da avaliação dos problemas } \\
\text { alimentares na rotina clínica, } \\
\text { considerando também as } \\
\text { características singulares dos } \\
\text { pacientes. }\end{array}$ \\
\hline $\begin{array}{l}\text { Fernandes et al. } \\
\qquad(2016)\end{array}$ & $\begin{array}{l}\text { Relatar a experiência vivenciada } \\
\text { diante da observação do } \\
\text { comportamento alimentar do } \\
\text { autista em um Centro Integrado } \\
\text { de Educação Especial da cidade } \\
\text { de Teresina, Piauí. }\end{array}$ & Profissionais da saúde & $\begin{array}{l}\text { O autismo é uma síndrome que } \\
\text { compromete todo o } \\
\text { desenvolvimento da criança e } \\
\text { adolescente, e por esse motivo } \\
\text { necessita de uma atenção } \\
\text { especial do poder público, } \\
\text { profissionais da saúde, } \\
\text { familiares e demais pessoas } \\
\text { envolvidas. }\end{array}$ \\
\hline $\begin{array}{l}\text { Lázaro \& Pondé } \\
\text { (2017) }\end{array}$ & $\begin{array}{l}\text { Investigar o comportamento } \\
\text { alimentar de indivíduos } \\
\text { com Transtorno do Espectro do } \\
\text { Autismo (TEA) através das } \\
\text { narrativas. }\end{array}$ & 18 mães & $\begin{array}{l}\text { Os profissionais devem instruir } \\
\text { os pais sobre o seu papel } \\
\text { decisivo no reforço ou } \\
\text { desencorajamento } \\
\text { comportamento inapropriado } \\
\text { nas refeições em crianças com } \\
\text { TEA. }\end{array}$ \\
\hline
\end{tabular}




\begin{tabular}{|c|c|c|c|}
\hline Gray et al. (2017) & $\begin{array}{l}\text { Investigar os comportamentos } \\
\text { durante as refeições de crianças } \\
\text { sino-americanas } \\
\text { Transtorno do Espectro do } \\
\text { Autismo (TEA). }\end{array}$ & $\begin{array}{l}31 \text { pais de crianças sino- } \\
\text { americanas com TEA. }\end{array}$ & $\begin{array}{l}\text { Estes achados podem fornecer } \\
\text { informações significativas aos } \\
\text { profissionais. }\end{array}$ \\
\hline $\begin{array}{l}\text { Gray et al. } \\
(2018)\end{array}$ & $\begin{array}{l}\text { Examinar os comportamentos } \\
\text { problemáticos durante as } \\
\text { refeições entre crianças com } \\
\text { Transtorno do Espectro do } \\
\text { Autismo (TEA) na Flórida. }\end{array}$ & 41 pais & $\begin{array}{l}\text { Os resultados indicam que } \\
\text { intervenções na primeira } \\
\text { infância são garantidas e mais } \\
\text { pesquisas em adolescentes são } \\
\text { necessárias. }\end{array}$ \\
\hline $\begin{array}{l}\text { Christensen et al. } \\
\qquad(2019)\end{array}$ & $\begin{array}{lr}\text { Revisar criticamente a literatura } \\
\text { existente sobre a possível } \\
\text { associação } & \text { entre } \\
\text { comportamentos } & \text { autistas e } \\
\text { comportamentos } & \text { alimentares } \\
\text { problemáticos em } & \text { populações } \\
\text { não clínicas. } & \end{array}$ & $\begin{array}{l}365 \text { crianças e adolescents } \\
\text { com TEA. }\end{array}$ & $\begin{array}{l}\text { A literatura existente indica } \\
\text { associações concorrentes entre } \\
\text { comportamentos autistas } \\
\text { específicos e comportamentos } \\
\text { alimentares problemáticos em } \\
\text { amostras não clínicas ao longo } \\
\text { das idades. }\end{array}$ \\
\hline $\begin{array}{l}\text { Margari et al. } \\
\quad(2020)\end{array}$ & $\begin{array}{l}\text { Destacar as evidências mais } \\
\text { recentes sobre epidemiologia e } \\
\text { apresentações de comer e comer } \\
\text { anormalidades comportamentais } \\
\text { emTEA desde a infância até a } \\
\text { adolescência. }\end{array}$ & $\begin{array}{l}\text { Crianças e adolescents } \\
\text { com TEA. }\end{array}$ & $\begin{array}{l}\text { Futuras investigações sobre as } \\
\text { implicações do perfil } \\
\text { comportamental, prejuízo } \\
\text { cognitivo, gravidade do TEA, } \\
\text { idade, e sexo na alimentação e } \\
\text { comportamentos na hora das } \\
\text { refeições são necessários, para } \\
\text { entender melhor os } \\
\text { mecanismos. }\end{array}$ \\
\hline $\begin{array}{l}\text { Yeung et al. } \\
\text { (2021) }\end{array}$ & $\begin{array}{l}\text { Comparar os comportamentos } \\
\text { durante as refeições e a } \\
\text { qualidade da dieta em pré- } \\
\text { escolares chineses com } \\
\text { Transtorno do Espectro do } \\
\text { Autismo (TEA) e em pré- } \\
\text { escolares com desenvolvimento } \\
\text { típico em Hong Kong. }\end{array}$ & $\begin{array}{l}65 \text { famílias com pré- } \\
\text { escolares com TEA de } 3 \text { a } \\
6 \text { anos e } 65 \text { famílias com } \\
\text { pré-escolaresr com } \\
\text { desenvolvimento típico } \\
\text { pareados por idade e sexo. }\end{array}$ & $\begin{array}{l}\text { Necessidade de monitoramento } \\
\text { regular e identificação precoce } \\
\text { de problemas comportamentais } \\
\text { e nutricionais durante as } \\
\text { refeições entre pré-escolares } \\
\text { com TEA. }\end{array}$ \\
\hline
\end{tabular}

Fonte: Dados da Pesquisa (2021).

Observa-se no Quadro 1 que os problemas comportamentais durante as refeições como recusa e seletividade alimentar podem ser observados assim como possuem um impacto negativo na saúde dessa população.

Kral et al. (2014) compararam o comportamento alimentar de crianças com Transtornos do Espectro do Autismo (TEA) e crianças com desenvolvimento típico (TDC), sendo que as crianças com TEA demonstraram um comportamento de maior evasão alimentar, relutância em comer alimentos significativamente maiores, seletividade sobre diversos alimentos já aceitos, além de uma alimentação insuficiente devido as emoções negativas. As crianças com sensibilidade sensorial atípica, que estão relacionadas a alterações sensoriais como a intolerância a certas texturas e cores de alimentos, estão em maior risco de comportamentos de esquiva de alimentos, e requerem suporte adicional em vários âmbitos da alimentação, com a finalidade de uma intervenção nutricional para ampliar os alimentos consumidos, além de uma educação nutricional para essas famílias, evitando desequilíbrios nutricionais, sendo portanto de grande valia um diagnóstico precoce da doença.

Sanches et al. (2015) demostraram que as crianças e adolescentes com TEA costumam escolher alimentos de alta densidade energética, como sucos, bebidas e lanches açucarados, fazendo baixo consumo de frutas e vegetais, bem como peixes e laticínios, o que evidencia a importância do acompanhamento nutricional para evitar desequilíbrios nutricionais.

Castro et al. (2016) constataram que os pacientes com TEA consumiram mais calorias que o grupo controle, e houve limitação do repertório alimentar, assim como uma alta prevalência de ingestão inadequada de algumas vitaminas e minerais. A monotonia da variedade de alimentos e outras interrupções na hora das refeições, a preferência por alimentos ricos em 
amido, lanches e uma baixa frequência do consumo de frutas, pode ocasionar uma limitação do repertório alimentar, e como consequência pode resultar em deficiências nutricionais.

Fernandes et al. (2016) relataram a experiência vivenciada diante da observação do comportamento alimentar do autista em um Centro Integrado de Educação Especial, constatando que as crianças se comportam de diversas formas. Foram observadas recusa ou preferência por certa cor, cheiro, consistência e textura do alimento, que manifestaram resistência para sentar-se à mesa e comer com outras pessoas e entrar no refeitório, assim como, para aceitar preparações novas.

Stough (2015) analisaram o comportamento de pais e filhos durante as refeições, associados ao consumo de alimentos desconhecidos por crianças com TEA, identificando que o único comportamento relacionado a comida desconhecida foi quando a criança deu uma mordida enquanto tomava goles da sua bebida, ao longo da refeição. Quando os pais incentivavam a criança a alimentar-se notou-se uma melhor aceitação da comida, aumentando a frequência de mordidas no alimento oferecido a criança, porém quando estas estavam distraídas e sem o incentivo dos pais, a frequência de mordidas no alimento foi mais baixa, notando-se que é imprescindível a participação dos pais durante a alimentação de crianças com TEA, sendo necessárias intervenções clinicas para a seletividade alimentar em crianças com TEA, fornecendo educação para os pais e estratégias durante as refeições, reduzindo os comportamentos inadequados das crianças durante as refeições.

Lázaro e Pondé (2017) evidenciaram que este se relaciona a fatores orgânicos como sensibilidade sensorial além da dificuldade em chupar e mastigar os alimentos, o que pode afetar diretamente em suas escolhas. Os fatores relacionados ao autismo podem afetar diretamente as escolhas alimentares dessas crianças. Sendo identificados os fatores ambientais, em particular o comportamento dos pais, o que pode desempenhar um papel decisivo, nas escolhas e incentivo a uma dieta mais diversificada.

Gray et al. (2017) evidenciaram que as crianças revelaram comportamentos problemáticos durante as refeições relatados pelos pais, como a preferência alimentos crocantes, não apresentavam disponibilidade para provar novos alimentos, e não permaneciam sentados a mesa até a refeição acabar. Por outro lado, as crianças não apresentavam comportamentos agressivos, ou perturbadores durante as refeições, quando comparadas a crianças norte americanas com TEA.

Gray et al. (2018) examinaram comportamentos problemáticos durante as refeições entre crianças com transtorno do espectro do autismo (TEA) relatados pelos pais, como a dificuldade para amamentar as crianças entre 3 e 6 anos, havia também a preocupação com a alimentação e dificuldades relacionadas a locais na hora das refeições, desejos e exigências por certos alimentos, diferente das crianças com comportamentos típicos, além disso constatou-se que havia diferenças de idade e dificuldades alimentares em casa, assim como para ingerir fast food e para se alimentar em restaurantes regulares.

Christensen et al., (2019) constataram que vários comportamentos autistas estão relacionados a problemas de comportamento alimentar, e o quociente do espectro do autismo, no qual se refere a extensão de traços autistas, sendo o conjunto de alterações do transtorno e a teoria da mente, mostram as associações mais fortes., nesse sentido mais estudos são necessários para compreender a ordem temporal dessas associações.

Margari et al., (2020) evidenciaram que o conhecimento atual não permite que sejam tiradas conclusões sobre o poder preditivo dos distúrbios alimentares quando reconhecidos na primeira infância. Consequentemente, estabelecendo o papel de comer e anormalidades de comportamento na hora das refeições como um marcador precoce de TEA. Dessa forma, futuras investigações sobre as implicações do perfil comportamental, prejuízo cognitivo, gravidade do TEA, idade, e sexo na alimentação e comportamentos na hora das refeições são necessários, para entender melhor os mecanismos além das dificuldades alimentares e fornecer informações adicionais úteis para desenvolver as estratégias de intervenção mais eficientes para problemas alimentares. 
Diante de tais comportamentos, principalmente relacionado a locais para alimentação, ressalta-se a importância do apoio e participação familiar durante as refeições dessas crianças, sendo de grande importância a intervenção nutricional nesse contexto, o que irá proporcionar uma melhor orientação aos pais e uma melhor alimentação para as crianças.

Yeung et al. (2021) compararam os comportamentos alimentares de crianças com desenvolvimento típico e crianças com TEA, sendo que as crianças com TEA apresentaram maiores pontuações de recusa alimentar, problemas com a alimentação no horário das refeições e menor qualidade e diversidade da dieta do que seus colegas com desenvolvimento normal.

\section{Considerações Finais}

Essas crianças reproduzem tanto padrões alimentares como comportamentos parecidos na hora das refeições, onde os pais desenvolvem um papel de grande importância nesse contexto, ficando evidente a gama de comportamentos e padrões realizados com frequência por crianças com TEA, assim como a maioria dos estudos sugerem que são necessárias intervenções profissionais para se criar novas estratégias na busca do enfrentamento de tal problemática. Constatou-se também, que a família é capaz de desenvolver comportamentos que podem auxiliar nesse processo educativo das crianças com TEA.

Diante do exposto, ressalta-se a relevância de um estudo abordando padrões e comportamentos alimentares de crianças com TEA, o que poderá auxiliar os pais e cuidadores que necessitam de uma atenção especial nesse âmbito, assim como a importância de intervenções da nutrição, desde que os aspectos nutricionais são imprescindíveis para uma boa saúde e qualidade de vida. Portanto, mais estudos transversais, longitudinais e de coorte voltados para esse grupo populacional são necessários, pois o mesmo é escasso na literatura, nesse sentido, o aconselhamento e acompanhamento dos pais de crianças com TEA trarão resultados positivos para a alimentação e entendimento desses comportamentos, buscando apresentar mais soluções acerca do tema apresentado e contribuindo com profissionais da área.

\section{Referências}

Bandini, L. et al. (2017). Changes in food selectivity in children with autism spectrum disorder. J Autism Dev Disord, 47(2): 439-446.

Carvalho, P. H. B. et al. (2013). Checagem corporal, atitude alimentar inadequada, insatisfação com a imagem corporal de jovens universitários. J. bras. psiquiatr. 62(2): 108-114.

Castro, L. et al. (2016). Feeding behavior and dietary intake of male children and adolescents with autism spectrum disorder: a case-control study. Int $J$ Dev Neurosci. 53(1): 68-74.

Costa, L. C. et al. (2020). Avaliações de distúrbios gastrointestinais nutricionais no transtorno do espectro autista: uma revisão integrativa. Res., Soc. Dev. 9(11): e169119498.

Christensen, S.S. et al. (2019). Disordered eating behaviors and autistic traits - is there any association in non-clinical populations? A systematic review Eur Eat Disord Rev. 27 (1): 8-23.

Fernandes, M. A. et al. (2016). Comportamento alimentar de crianças e adolescentes autistas atendidas em um centro integrado de educação especial. Rev Enferm UFP, 5(1):101-104.

Gray, H. L. et al. (2018). Early history, mealtime environment, and parental views on mealtime and eating behaviors among children with ASD in Florida. Nutrients, 10: 1852-1867.

Gray, L. H. et al. (2017). Mealtime behaviors of Chinese american children with Autism Spectrum Disorder. J Autism Dev Disord. 47 (3): $892-897$.

Gomes, A. B. G. et al. (2020). Transtorno do espectro autista e hábito alimentar de crianças e adolescentes Res., Soc. Dev, 9(10): e7319108978.

Gomes, V. T. S. et al. (2016). Nutrição e autismo: reflexões sobre a alimentação do autista. XX Encontro Latino Americano de Iniciação Científica, XVI Encontro Latino Americano de Pós-Graduação e VI Encontro de Iniciação à Docência - Universidade do Vale do Paraíba.

Kral, T. V. E. et al. (2014). Child eating behaviors and caregiver feeding practices in children with Autism Spectrum Disorders. Publ Health Nurs, 32 (5): 48897.

Lázaro, C. P., \& Pondé, M. P. (2017). Narratives of mothers of children with autism spectrum disorders: focus on eating behavior. Am J Psychother, 39(3): 180-187. 
Research, Society and Development, v. 10, n. 12, e296101219911, 2021

(CC BY 4.0) | ISSN 2525-3409 | DOI: http://dx.doi.org/10.33448/rsd-v10i12.19911

Margari, L. et al. (2020) Eating and Mealtime Behaviors in Patients with Autism Spectrum Disorder: Current Perspectives. Neuropsychiatr. Dis. Treat. 16: 2083- 2102

Pintos, R.N. et al. (2016). Autismo infantil: impacto do diagnóstico e repercussões nas relações familiares. Rev. Gaúcha Enferm. .37(3): e61572.

Rocha, G. S. S. et. al. (2019). Análise da seletividade alimentar de crianças com Transtorno do Espectro Autista. REAS. Revista Eletrônica Acervo Saúde. 24: e538.

Sánchez, S. S. et al. (2015). Selectividad alimentaria en los trastornos del espectro autista: una revisión sistemática. Rev Esp Nutr Comunitaria; $21(2): 13-19$.

Stough, C. O. (2015). Mealtime behaviors associated with consumption of unfamiliar foods by young children with autism spectrum disorder. Appetite, 95: $324 \mathrm{e} 333$.

Sousa, L. M. M. S., Marques-Vieira, C. M. A., Severino, S. S., \& Antunes, A. V. (2017). Metodologia de revisão integrativa da literatura de enfermagem. Revista Investigação em Enfermagem. 21(2): 17-26.

Westwood, H. et al. (2017). Clinical evaluation of autistic symptoms in women with anorexia nervosa. Molecular Autism, 8:(12) 01-9.

Yeung, S.S. et al. (2021). Eating behaviors and diet quality in Chinese preschoolers with and without autism spectrum disorder: a case-control study. $J$ Pediatr. 21(5): 1-20. 